Meta

Journal des traducteurs

Translators' Journal

\title{
ANTIA, BASSEY EDEM (2007) : Indeterminacy in Terminology and LSP. Amsterdam et Philadelphie : John Bejamins, 233 p.
}

\section{Mélanie Labelle}

Volume 56, numéro 1, mars 2011

URI : https://id.erudit.org/iderudit/1003521ar

DOI : https://doi.org/10.7202/1003521ar

Aller au sommaire du numéro

\section{Éditeur(s)}

Les Presses de l’Université de Montréal

\section{ISSN}

0026-0452 (imprimé)

1492-1421 (numérique)

Découvrir la revue

Citer ce compte rendu

Labelle, M. (2011). Compte rendu de [ANTIA, BASSEY EDEM (2007) :

Indeterminacy in Terminology and LSP. Amsterdam et Philadelphie : John

Bejamins, 233 p.] Meta, 56(1), 219-220. https://doi.org/10.7202/1003521ar

Ce document est protégé par la loi sur le droit d'auteur. L'utilisation des services d'Érudit (y compris la reproduction) est assujettie à sa politique d'utilisation que vous pouvez consulter en ligne.

https://apropos.erudit.org/fr/usagers/politique-dutilisation/ 
entraîner l'annulation de l'acte en cause par le juge administratif» (p. 230).

Ainsi, ce n'est pas en cherchant une information précise, mais en butinant dans ce dictionnaire que le traducteur d'ici y trouvera son compte. Par exemple, comment ne pas être intrigué par cette notion de fongibilité d'un budget? ("Caractéristique de crédits budgétaires dont l'objet et la nature ne sont précisés qu'à titre indicatif, le gestionnaire de ces crédits pouvant les redéployer en cours d'exercice sous certaines conditions afin d'optimiser sa gestion» [p. 229]). Encore là, la question se pose: le mot est beau, mais quand sera$\mathrm{t}$-il opportun de l'utiliser? Idem pour infogérance («Technique visant à externaliser tout ou partie de l'informatique et à la confier à un prestataire. » [p. 254]). Une fois encore, c'est peut-être plus la formulation de la définition que son contenu qui sera utile au traducteur.

Au-delà de ces réserves, il y a lieu de signaler une section d'une dizaine de pages par laquelle les auteurs font preuve d'une originalité digne de mention et tombent, cette fois, pile-poil sur les attentes du traducteur. Il s'agit de l'article Verbes de l'administration, qui sort du domaine de prédilection habituel des auteurs de ce genre d'ouvrage - le substantif - pour présenter quelques dizaines de verbes typiques du langage administratif, avec exemples d'utilisation à l'appui et souvent cooccurrents, depuis alléger des charges, arrêter (au sens de /fixer/, /décider/) et asseoir sur (/ calculer sur une base déterminée/) jusqu'à stipuler et surseoir, en passant par fonder en droit, grever, homologuer...

Bref, un outil plein de petits trésors, pour peu qu'on soit conscient des limites inévitables que présente un ouvrage sur le français de l'administration hexagonale pour un traducteur canadien.

François LavalléE Magistrad, Québec, Canada

\section{Antia, BAssey Edem (2007): Indeterminacy in Terminology and LSP. Amsterdam et Phila- delphie: John Bejamins, 233 p.}

Indeterminacy in Terminology and LSP, publié en hommage à Heribert Picht, rassemble des articles de plusieurs auteurs explorant la problématique de l'indétermination selon différentes perspectives. Peu importe leur point de vue, les auteurs s'accordent sur le fait que l'indétermination est un sujet à considérer dans le cadre d'études en terminologie et en langues de spécialité (LSP). L'intérêt pour cette question est relativement récent, car elle va à l'encontre des principes de la terminologie dite classique.
Cet ouvrage est divisé en quatre sections, en fonction du point de vue selon lequel le sujet est abordé. Les articles de la première section traitent de l'indétermination dans une perspective lexicale. Ceux de la deuxième touchent plutôt les aspects épistémologiques de la question. La troisième section concerne les enjeux soulevés par le problème de l'indétermination pour la modélisation en terminologie et en LSP. Dans la dernière section, les contributions d'Heribert Picht en terminologie et en LSP sont exposées.

Dans la première partie, on trouve d'abord l'article de Øivin Andersen, professeur de linguistique en Norvège. Dans son article, il contraste les concepts d'indétermination et de formation adéquate (well-formedness) ainsi que les concepts d'imprécision et d'exactitude. Par la suite, Margaret Rogers, professeure de traduction et de terminologie et directrice du Centre for Terminological Studies de l'Université de Surrey au RoyaumeUni, traite de l'indétermination d'un point de vue lexical dans le contexte de la traduction. Elle y remarque entre autres qu'en traduction, il existe une indétermination dans le réseau d'équivalences entre les unités lexicales participant à la chaîne lexicale, mais que la majorité des paires d'équivalents sont déterminées. Puis, Sergej Griniewicz, professeur de linguistique en Russie, présente une étude dans le domaine de l'anthropologie linguistique. Selon lui, l'indétermination est toujours présente et les changements correspondent aux stades de l'évolution humaine. Anciennement, les limites de la signification des mots n'étaient pas clairement établies, mais ce qui est perçu comme vague aujourd'hui ne l'était probablement pas. Le dernier article de cette partie est de Klaus-Dirk Schmitz, de Cologne en Allemagne. Il porte sur le lien entre l'indétermination et l'évaluation d'un logiciel par l'utilisateur dans une perspective de gestion de la terminologie et de localisation. Il contraste l'imprécision (le référent fait partie du concept) et l'ambiguïté (plus d'une interprétation possible d'un concept).

L'article de Gerhard Budin, qui est notamment professeur et directeur du Département de traduction à l'Université de Vienne, débute la deuxième partie de l'ouvrage. Une perspective épistémologique y est adoptée pour présenter l'évolution du concept de l'indétermination dans certaines disciplines scientifiques. Une importance particulière est accordée à la caractérisation du postmodernisme, notamment à l'aide de la présentation de quelques oppositions. En conclusion, les considérations propres à la terminologie sont présentées. Vient ensuite la contribution de Johan Myking, du Département des langues et de la littérature scandinaves à Bergen en Norvège, qui traite des critiques de la terminologie classique. Ces 
critiques partent de deux dichotomies présentes dans la terminologie classique: la distinction entre la communication spécialisée et la communication générale et la distinction entre le terme et les autres unités lexicales. Vladimir Leitchik de Moscou présente, quant à lui, différentes définitions en s'attardant à leur degré d'indétermination. Il existe plusieurs types de définition (générique-spécifique, énumérative, contextuelle, opérationnelle). Certains types de définition fonctionnent comme des dichotomies, c'est-à-dire que le terme correspond à la définition ou non, comme en mathématiques, alors que d'autres permettent plusieurs interprétations, elles sont dans une certaine mesure floues. Suit l'article de Birthe Toft, professeure associée de l'University of Southern Denmark au Danemark. Elle y examine le concept d'équilibre en économie, d'un point de vue des sciences cognitives expérientielles à partir du discours de divers économistes. Elle compare d'abord la position de la terminologie classique par rapport aux concepts. Elle conclut par une discussion de l'indétermination dans la langue de l'économie classique et moderne. Ingrid Simonnaes, professeure au Norwegian School of Economics and Business Administration (NHH), se penche sur la notion d'indétermination par rapport aux termes juridiques. L'article se place dans la perspective de la théorie moderne de la terminologie. La notion de concept, elle-même vague, y est tout d'abord discutée, de même que la différence entre la signification et le concept. Selon elle, les concepts sont et doivent être vagues, ce qui ne signifie par contre pas qu'ils sont ambigus. Le dixième chapitre, qui conclut la deuxième section, par Reiner Arntz de l'Université de Hildesheim en Allemagne et Peter Sandrini de l'Université de Innsbruck en Autriche, n'a pas été traduit de l'allemand. Leur article traite de l'indétermination de la langue juridique en différentes langues dans une optique d'amélioration de la terminologie multilingue et de la traduction.

La troisième section s'ouvre par la contribution de Sue Ellen Wright (Kent State University). Elle est professeure de traduction et s'intéresse entre autres à la terminologie et à la localisation. Dans cet article, elle tente de réconcilier deux positions concernant l'indétermination: la position des linguistes qui ont accepté la présence d'indétermination et celle des informaticiens qui la refusent. Ces deux visions se rencontrent notamment dans les systèmes d'organisation des connaissances (knowledge organisation system) ou les systèmes de représentation des connaissances (knowledge representation resource). Ensuite, Bodil Nistrup Madsen de Copenhague au Danemark, professeure dans le domaine des langues et de la terminologie, traite de l'indétermination en lien avec les ontologies, plus précisément avec le projet
Computer-Aided Ontology Structuring (CAOS). Certains principes y sont utilisés pour diminuer l'indétermination: dimensions uniques, caractéristiques principales uniques et groupement par sous-dimensions. Elle y remarque également que l'indétermination n'existe pas dans une ontologie, mais qu'elle provient plutôt de la comparaison de différentes possibilités d'organisation des connaissances. Le dernier article est signé Anita Nuopponen (Vaasa, Finlande), qui est une des pionnières de la recherche en terminologie en Finlande. Dans son article, il est question des processus de modélisation permettant de présenter différentes relations entre les concepts. Cette étude s'inscrit dans le cadre d'un projet visant à adapter des méthodes terminologiques d'analyse de concept pour des spécialistes. La difficulté à définir les concepts centraux y est exposée.

En conclusion, cet ouvrage constitue une bonne introduction à la question de l'indétermination en langue de spécialité. Les diverses perspectives qui y sont adoptées ainsi que les nombreux domaines étudiés permettent de faire un survol de cette problématique sous plusieurs angles. De plus, le fait de tenir compte de l'indétermination en terminologie et dans l'étude des LSP permet de renouveler le domaine en s'éloignant de la terminologie dite classique.

Mélanie Labelle Montréal, Canada

Merrill, Christi (2009): Riddles of Belonging. India in Translation and Other Tales of Possession. New York: Fordham University Press, 380 p.

This is surely one of the most original, and potentially influential, books in translation studies to have appeared in many years. Written with spirit, it shares the verbal virtuosity and playfulness of writers like Doris Sommer (Bilingual Aesthetics) and Suzanne Jill Levine (The Subversive Scribe). These are books that venture into heavyweight issues with a light touch, and all the more effectively leave their imprint. Riddles of Belonging takes on issues of historical inequality, human rights and global justice through a series of interlocking stories. In this highly inventive book, concepts spiral through the narrative, acquiring new meanings as they are approached from different angles.

Christi Merrill combines a range of qualities and aptitudes unusual to find in one individual. She is a scholar at ease with the most recent developments in postcolonial theory and translation studies. She is also an accomplished translator from Hindi and Rajasthani, and she is a gifted writer. That she uses these overlapping skills to 